\title{
Perfluorooctanoic acid rigidifies a model lipid membrane
}

\author{
B. Brüning ${ }^{1,2}$ and B. Farago ${ }^{3}$ \\ ${ }^{1}$ Soft Matter and Functional Materials, Helmholtz-Zentrum Berlin, Berlin, Germany \\ ${ }^{2}$ Reactor Institute Delft, Delft University of Technology, Delft, The Netherlands \\ ${ }^{3}$ Time-of-Flight and High Resolution, Institut Laue-Langevin, Grenoble, France
}

(Received 9 March 2014; published 17 April 2014)

\begin{abstract}
We report a combined dynamic light scattering and neutron spin-echo (NSE) study on vesicles composed of the phospholipid 1,2-dimyristoyl-sn-glycero-3-phosphatidylcholine under the influence of varying amounts of perfluorooctanoic acid. We study local lipid bilayer undulations using NSE on time scales up to $200 \mathrm{~ns}$. Similar to the effect evoked by cholesterol, we attribute the observed lipid bilayer stiffening to a condensing effect of the perfluorinated compound on the membrane.
\end{abstract}

DOI: 10.1103/PhysRevE.89.040702

Perfluorinated compounds (PFCs) are fully fluorinated fatty acid analogs commonly used in a wide range of applications, such as the production of fire-extinguishing foams, anticorrosion agents, lubricants, or cosmetics [1,2]. As a consequence of their chemical stability, these compounds exhibit an environmental stability and are transmitted into the mammal food chain [3]. Due to their tendency to bioaccumulate, the compounds affect properties of cell membranes, causing developmental and reproductive disorders [2,4]. It is of particular interest to understand the effect of perfluorinated compounds not only on cellular membranes [5,6], but also on their biomimetic counterparts [7]. In mammal organisms, vesicular membranes often serve as natural carriers. It is assumed that functional properties of a membrane depend likewise on its composition-dependent structure and dynamics $[8,9]$. In order to gain insight into membrane function, specific material properties, such as, e.g., the bilayer bending rigidity $\kappa$ can be aimfully influenced. Several studies have addressed the insertion of perfluorinated compounds into binary model membranes. Oriented mono- and bilayers were investigated by Matyszewska et al. using methods including surface pressure and potential measurements, infrared spectroscopy (IR), nuclear magnetic resonance (NMR) techniques, and molecular dynamics (MD) simulations [4,10,11]. While the phospholipid head group tilt against the bilayer normal decreases with rising amounts of inserted perfluorooctanoic acid (PFOA), the lipid acyl chain order increases. Lateral molecule diffusion in the membrane plane changes nonuniformly as the ratio of the components in the binary mixture is varied [10]. Lehmler et al. study liposomes containing binary mixtures of varying phospholipids and perfluorinated surfactants [12-15]. They use fluorescence spectroscopy and differential scanning calorimetry measurements to study the partitioning of surfactants into the phospholipid bilayer and find that it is independent of the lipid acyl chain length. While the phase behavior is largely independent of the type of phospholipid, PFOA itself is found to partition more readily into lipid bilayers in their fluid phase [13]. Several studies have taken advantage of a combination of dynamic light scattering (DLS) and long-wavelength NSE for the investigation of the local bilayer undulation dynamics in unilamellar lipid vesicles (ULVs) [16-21]. Here, we cover a window of more than 200 ns. The membrane dynamics was investigated by NSE using wavelengths of $\lambda=10$ and $17 \AA$. We highlight comple-
PACS number(s): 87.85.jj, 82.70.Uv, 68.35.Ja, 29.30.Hs

mentary dynamic regimes by adapting the time range of the correlation function decay. We use our DLS results for a quantitative separation of two dynamical processes in the $17 \AA$ data.

The phospholipid 1,2-dimyristoyl-sn-glycero-3phosphatidylcholine (DMPC) was purchased from Avanti (Alabaster, AL), and the perfluorinated surfactant PFOA from Sigma-Aldrich (Steinheim, Germany). Components were dissolved in chloroform in the desired molar proportions. The solvent was evaporated and the dry lipids were hydrated with heavy water $\left(\mathrm{D}_{2} \mathrm{O}\right)$ at $10 \mathrm{mg} / \mathrm{ml} . \mathrm{D}_{2} \mathrm{O}$ was used both for dynamic light scattering (DLS) and neutron spin echo (NSE). For each specific composition, the $p \mathrm{H}$ was compensated through the addition of a $\mathrm{Na}_{2} \mathrm{CO}_{3}$ base. To obtain unilamellar vesicles (ULVs), the suspension was passed ten times through a polycarbonate filter with a $500 \AA$ pore diameter using a LiposoFast Basic Extruder (Avestin, Canada). For the NSE experiment, samples were poured into $1 \mathrm{~mm}$ thick quarz cuvettes with a quadratic cross section of $35 \mathrm{~mm}$ by $35 \mathrm{~mm}$ (Hellma, Müllheim, Germany).

For dynamic light scattering (DLS), an ALV goniometer with a $35 \mathrm{~mW}$ He-Ne laser operating at a wavelength of $632.8 \mathrm{~nm}$ was used with an ALV/high quantum efficiency (QE) avalanche photodiode (APD) detector and an ALV-6010/160 external multiple $\tau$ digital correlator unit. The vesicle centerof-mass diffusion can be described by a correlation function $g_{1}(t)=\exp \left(-D q^{2} t\right)$, which is derived from the measured intensity correlation function $g_{2}(t)$ through the Siegert relation $g_{1}(t)=\sqrt{g_{2}(t)-1}$. The center-of-mass diffusion coefficient $D$ and the hydrodynamic vesicle radius $R_{H}$ are linked according to the Stokes-Einstein equation $R_{H}=\frac{k_{B} T}{6 \pi \eta(T) D} \cdot k_{B}$ denotes the Boltzmann constant, $T$ the absolute temperature, and $\eta(T)$ the temperature-dependent solvent viscosity $\left[\right.$ for $\mathrm{D}_{2} \mathrm{O}$ at $30^{\circ} \mathrm{C}$, $\left.\eta(T)=0.973 \times 10^{-3} \mathrm{~Pa} \mathrm{~s}\right]$. Long-term measurements indicate that the averaged vesicle radii $R_{H}$ remained constant for at least a week. The vesicles for the combined DLS and NSE experiment were freshly prepared and measured within 1 day. Angle-dependent measurements were taken between $20^{\circ}$ and $150^{\circ}$ in steps of $10^{\circ}$; the obtained relaxation rates $\Gamma_{d}$ vs $q^{2}$ are shown in Fig. 1. Linear fits indicate a purely Fickian diffusion. The linear slope of these curves corresponds to the vesicle center-of-mass diffusion constant $D$.

The neutron spin-echo (NSE) experiment was performed at the cold spectrometer IN15 at the Institut Laue Langevin (ILL, Grenoble, France). Due to its fine angular resolution 


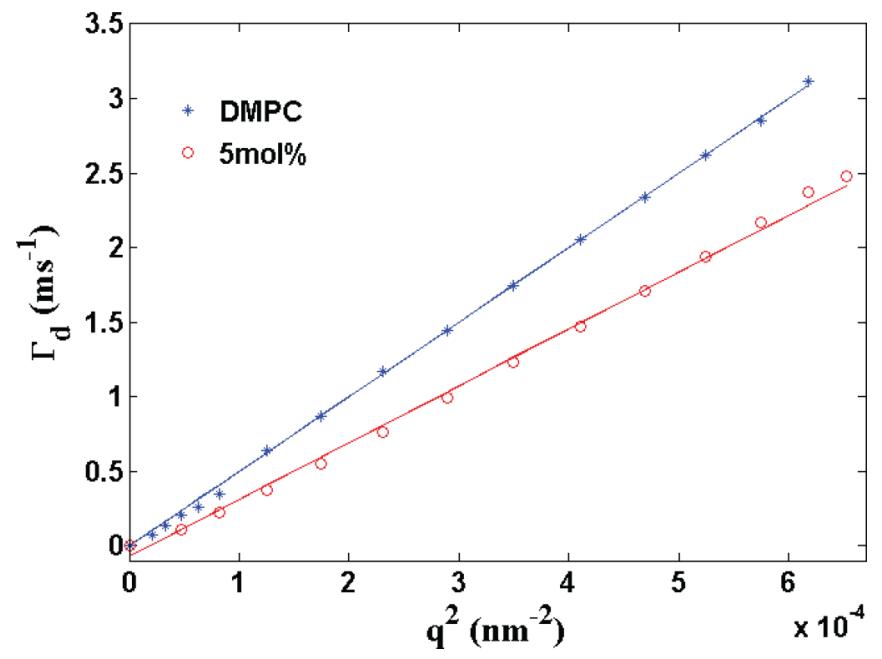

FIG. 1. (Color online) Relaxation rate $\Gamma_{d}$ vs $q^{2}$ for vesicles composed of DMPC and DMPC/PFOA (5 mol \%). Linear fits yield the vesicle center-of-mass diffusion constant $D$.

in the small-angle regime (small $q$ ), the instrument is well suited to probe mesoscopic length scales. An introduction into the NSE method is given in Ref. [22]. A measurement yields a momentum transfer and time-resolved intermediate scattering function $S(q, t)$, in which the Fourier time $t$ changes proportionally to the wavelength $\lambda^{3}$ and the applied magnetic field integral, following $t \propto \lambda^{3} \int|B| d l$. Error bars in the measured data correspond to $\pm \sigma$ statistical error calculated from the counting statistics and are transferred to our least squares fits. The $q$ range probed by NSE lies in the range of the inverse length scales of local bilayer interface undulations. This allows a data interpretation on the basis of models including a unique $q$ dependence of the measured relaxation rates $\Gamma(q)$. On IN15, incident neutron wavelengths between 6 and $25 \AA$ with a wavelength spread of $15 \%$ are available. At a distance of $4.6 \mathrm{~m}$ from the sample, a ${ }^{3} \mathrm{He} / \mathrm{CF}_{4}$ multidetector is located with $32 \times 32$ pixels of $1 \mathrm{~cm}^{2}$ each. The instrumental resolution is determined using graphoil as a purely coherent elastic scatterer and accounted for by division of sample scattering through the resolution signal [23].

Merely simple diffusion mechanisms were assumed for the short wavelength data $S(q, t) \propto \exp \left(-\Gamma_{f} t\right)$, with a relaxation rate $\Gamma_{f}=D_{f} q^{2}$. An example is shown in Fig. 2(a) for DMPC at $30{ }^{\circ} \mathrm{C}$, within the $q$ range in which the most pronounced changes in the dynamics occur. Figure 2(b) then shows an effective diffusion constant $D_{f}^{\text {eff }}(q)$ derived as $\Gamma_{f}(q) / q^{2}$ for DMPC and DMPC/PFOA (10 mol \%). In both cases, this plot is not $q$ independent, as would be the case if only Fickian diffusion occurred in the probed time range. Unfortunately, the different dynamic contributions cannot be unambiguously separated, due to a lack of a full polarization decay at the largest measured $q$ shown in Fig. 2(a). The dynamics are not fully covered within the probed window, necessitating our choice of a higher wavelength to extend the Fourier-time range. Here, a smaller $q$ range is covered with higher resolution, thus two complementary data sets are obtained. Regarding the origin of the quadratic mode $\Gamma_{f}\left(q^{2}\right)$, several possibilities exist: In their seminal theoretical
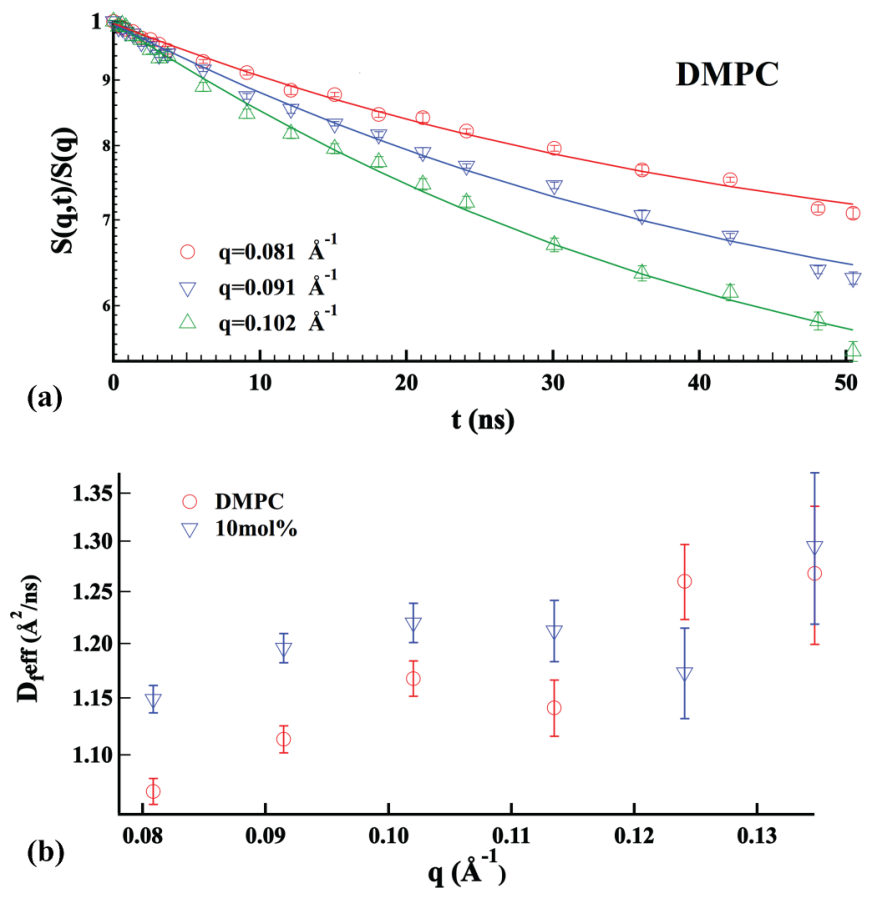

FIG. 2. (Color online) (a) Normalized intermediate scattering function $S(q, t) / S(q)$ for DMPC $\left(30{ }^{\circ} \mathrm{C}\right)$ at $\lambda=10 \AA$. Singleexponential fits yield the relaxation rates $\Gamma_{f}(q)$; (b) the effective diffusion constant $D_{f}^{\text {eff }}(q)=\Gamma_{f}(q) / q^{2}$ exhibits a $q$ dependence, indicating further dynamics.

works, Evans and Yeung, as well as Seifert and Langer, have already predicted modes which relate bending and local density changes between the two monolayers and have taken into account the resulting intermonolayer friction [24,25]. The modes follow quadratic relations $\Gamma\left(q^{2}\right)$, and have been discussed in recent experimental works by Arriaga et al. [18]. On the other hand, the contribution lies close to the one of the vesicle center-of-mass diffusion found by dynamic light scattering. Following, we discuss vesicle center-of-mass translations and bilayer undulations as separable dynamic contributions in the long-wavelength spin-echo data.

Curvature undulations of elastic membranes are commonly described by the well-known Helfrich Hamiltonian [26]. Based on this continuum mechanical approach, Milner and Safran further describe the fluctuation dynamics of microemulsion droplets and vesicles [27]. In their theory, the normal bending modes of the flexible interface are coupled to the viscous friction exerted by the suspending medium according to a single-exponential decay $\exp \left(-\Gamma_{\mathrm{MS}} t\right)$ with a relaxation rate $\Gamma_{\mathrm{MS}}=\frac{\kappa}{4 \eta} q^{3}$, where $\eta$ is the effective viscosity of the solvent medium and $\kappa$ the bilayer bending rigidity. Faster relaxations are assigned to stiffer membranes. While suited to describe soft interfaces with bending rigidities on the order of $k_{B} T$, such as microemulsion droplets and sponge phases, the expression fails to accurately account for the dynamics of model lipid membranes with bending rigidities of several $k_{B} T$. Zilman and Granek describe curvature shape fluctuations of freely suspended phospholipid bilayers [28,29]. Their model takes into account a coupling of the bending modes and local diffusion processes: In a rigid membrane with a bilayer bending rigidity of $\kappa \gg k_{B} T$, less free volume can 
be explored by single molecules; this means that a relaxation rate for a coupled process of undulation and local curvature will increase, whereas the average amplitude of the modes will decrease. The anomalous subdiffusive relaxation of the bending motions is described by a stretched exponential decay with a stretching exponent of $\beta=2 / 3$ :

$$
S(q, t) \propto \exp \left[-\Gamma_{u}(q) t\right]^{\beta},
$$

with

$$
\Gamma_{u}(q)=0.025 \gamma_{q}\left(\frac{k_{B} T}{\kappa}\right)^{1 / 2}\left(\frac{k_{B} T}{\eta(T)}\right) q^{3} .
$$

The relaxation rate $\Gamma_{u}(q)$ includes the temperature-dependent solvent viscosity $\eta(T)$. Further, $\gamma_{q}$ is a weak monotonous function of the bending rigidity $\kappa$ according to $\gamma_{q}=1-$ $\frac{3}{4 \pi}\left(\frac{k_{B} T}{\kappa}\right) \ln (q h)$, where $h$ is the membrane thickness with $q h \approx 1$. When $\kappa$ lies on the order of several $k_{B} T, \gamma_{q}$ can be approximated to unity. Using atomistic and coarse-grained molecular dynamics (MD) simulations, Brandt and Edholm describe the nanometer length scale fluctuation decay in fluid biomembranes by a stretched exponential [30]. We discuss our experimental data on the basis of this approach, augmented by the occurrence of underlying vesicle center-of-mass diffusion [Eq. (2)]. $A$ is a normalization parameter close to one, the mass diffusion relaxation rate $\Gamma_{d}=D q^{2}$ is fixed, the relaxation rate of bilayer undulations $\Gamma_{u}$ is a free parameter, and the stretched exponential is held to $\beta=0.66$, following the Zilman-Granek approach. With respect to previous works, such as Refs. [16,17], this seems to be a more general approach. Comparing the outcome to the results obtained on the basis of
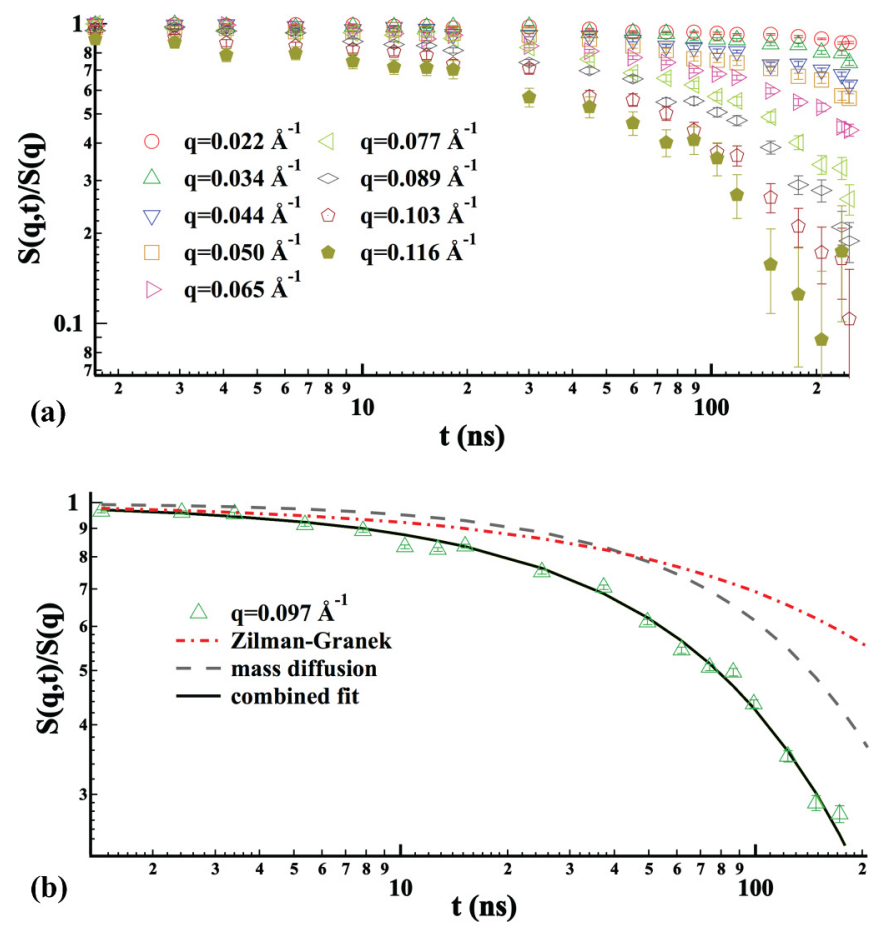

FIG. 3. (Color online) Normalized intermediate scattering functions $S(q, t) / S(q)$ for DMPC $\left(30^{\circ} \mathrm{C}\right)$ : (a) $q$ range covered at $\lambda=$ $17 \AA ̊$. (b) Combined fit according to Eq. (2); single contributions are indicated by dotted lines. the previous description of $S(q, t) / S(q)$, we find no significant differences within experimental precision:

$$
S(q, t) / S(q, 0)=A \exp \left(-\Gamma_{d} t\right) \exp \left(-\Gamma_{u} t\right)^{\beta} .
$$

The normalized intermediate scattering function of the DMPC standard $S(q, t) / S(q)$ is shown in Fig. 3(a) for varying $q$. Fourier times extend up to $200 \mathrm{~ns}$, and the normalized polarization nearly fully decays. This means the dynamics are captured within the spectrometer window, enabling more precise treatment than before. Over the whole $q$ range, fits can be improved taking into account dynamic contributions just outside the instrumental window in the $\mu$ s regime. Therefore, we add a vesicle center-of-mass diffusion contribution through the diffusion constant $D$ obtained from DLS measurements [Eq. (2)]. In Fig. 3(b), both vesicle center-of-mass diffusion as well as bilayer undulation contributions are shown for one exemplary $q$ value $(q=0.097 \AA)$. The double logarithmic scale shows that the decay curvature is well matched by the combined fit. At momentum transfers of $q=0.071 \AA$ and above, a deviation from the single-exponential behavior is observed, starting at Fourier times between 50 and $100 \mathrm{~ns}$ [cf. Fig. 3(a)]. The undulation relaxation rate $\Gamma_{u}(q)$ can now be further analyzed. For our DMPC standard in its fluid phase $\left(30{ }^{\circ} \mathrm{C}\right)$, the result of $\kappa=17.68 \pm 0.15 k_{B} T$ derived from linear regression of $\Gamma_{u}\left(q^{3}\right)$ is well in accordance with literature values [31-33]. The inherent experimental error for $\kappa$ is estimated to lie on the order of $k_{B} T$. The undulation relaxation rate $\Gamma_{u}\left(q^{3}\right)$ obtained from these fits is shown in Fig. 4. The insertion of rising amounts of PFOA evokes a decrease in the linear slope of $\Gamma_{u}\left(q^{3}\right)$. The resulting increase in the bilayer bending rigidities $\kappa$ is shown in Fig. 4(b).
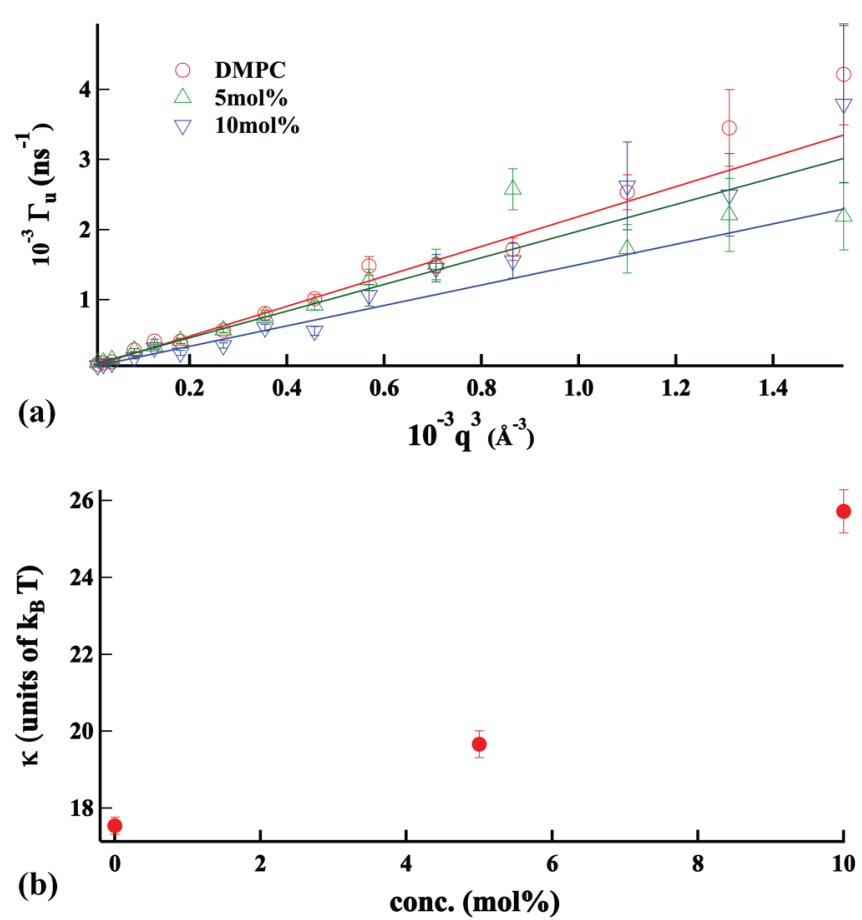

FIG. 4. (Color online) Bilayer undulations: (a) Relaxation rate $\Gamma_{u}$ vs $q^{3}$, for DMPC and DMPC/PFOA. Linear fits yield the concentration-dependent bilayer bending rigidity $\kappa$ shown in (b). 
Comparing unilamellar vesicles composed of DMPC and DMPC/PFOA mixtures, we have investigated the effect of the perfluorinated compound on local bilayer undulations and on the bilayer bending rigidity $\kappa$. Effects on vesicle self-diffusion were also investigated. The NSE data can be meaningfully analyzed, assuming a combination of two separable contributions within the dynamic window up to $200 \mathrm{~ns}$, namely, vesicle center-of-mass diffusion and local bilayer undulations. The latter was described on the basis of the well-known Zilman-Granek approach for free film fluctuations. Our data support a view that lipid bilayer undulation dynamics and corresponding bending rigidities $\kappa$ can be tuned by directly inducing changes at the lipid acyl chains: The perfluorinated compound PFOA, which partitions into the membrane [12-15], reduces the free volume in the membrane plane, since the $\mathrm{CF}_{2}$ segments of the surfactant tail occupy more space than the neighboring lipid chain $\mathrm{CH}_{2}$ segments. Consequently, an increase in the bilayer bending rigidity $\kappa$ is observed. In a previous work, we have also investigated binary lipid mixtures containing DMPC and the monounsaturated phospholipid 1,2-dioleoyl-sn-glycero-3-phosphatidylcholinel (DOPC) [19]. Here, a significant decrease in $\kappa$ was observed, the higher the amount of the latter was. We suggested this decrease might be explained by a mesoscopic lateral phase segregation leading to domain structure fluctuations and a corresponding softening of the membrane [34,35]. In the case of lipid/surfactant mixtures, on the other hand, a homogenous lateral distribution of the two components is likely, similar to the one in binary cholesterol mixtures. The stiffening of a DMPC bilayer through cholesterol insertion has long since been associated with the condensing effect of the sterol [36].
Nakahara et al. have suggested similar effects might occur for partially fluorinated alcohols in dipalmitoylphosphatidylcholine (DPPC) and dipalmitoylphosphatidylglycerol (DPPG) membranes [37]. In our case, the PFOA-induced stiffening of the DMPC bilayer might be explained as follows: The ionic surfactant head is more hydrophillic and the perfluorinated tail more hydrophobic than the zwitterionic phospholipid head group and its acyl chains. Repulsion between neighboring surfactant heads as well as the bulkiness of the surfactant $\mathrm{CF}_{2}$ segments in comparison to their $\mathrm{CH}_{2}$ lipid acyl chain counterparts make an alternating in-plane molecule distribution of lipid and surfactant likely. The rotation of lipid acyl chain segments around the bilayer normal axis is then restricted. Thus, by PFOA inclusion, the in-plane area density of lipid acyl chain and surfactant tail segments is then increased, causing lipid bilayer stiffening. Such a bilayer stiffening may well be the origin of the compounds' harmful effects on cellular membranes, as it is likely to influence the fusiogenic properties of lipid vesicles. Therefore, it would be interesting to study how the compounds influence the formation of stalk intermediates as precursor states for the fusion of lipid vesicles. This could further elucidate how reproductive and developmental processes are inhibited in mammal organisms after perfluorinated compound ingestion.

We are grateful to Ralf Köhler and Ralf Stehle for experimental support. Roland Steitz is thanked for helpful comments on the manuscript. We thank HZB for financial support and for the allocation of beam time on IN15, as well as ILL for technical support.
[1] E. Kissa, Fluorinated Surfactants and Repellents (Dekker, New York, 2001).

[2] J. G. Riess, Curr. Opin. Colloid Interface Sci. 14, 294 (2009).

[3] M. H. Ropers, S. Durand, B. Veyrand, V. Beaumal, P. Marchand, M. Anton, and B. L. Bizec, Food Hydrocolloids 23, 1149 (2009).

[4] D. Matyszewska, J. Leitch, R. Bilewicz, and J. Lipkowski, Langmuir 24, 7408 (2008).

[5] H. Nakahara and O. Shibata, J. Oleo Sci. 61, 197 (2012).

[6] M. P. Krafft, Adv. Drug Delivery Rev. 47, 209 (2001).

[7] A. Zaggia and B. Ameduri, Curr. Opin. Colloid Interface Sci. 17, 188 (2012).

[8] B. Brüning, M. C. Rheinstädter, A. Hiess, B. Weinhausen, T. Reusch, S. Aeffner, and T. Salditt, Eur. Phys. J. E 31, 419 (2010).

[9] M. C. Rheinstädter, J. Das, E. J. Flenner, B. Brüning, T. Seydel, and I. Kosztin, Phys. Rev. Lett. 101, 248106 (2008).

[10] D. Matyszewska, K. Tappura, G. Orädd, and R. Bilewicz, J. Phys. Chem. B 111, 9908 (2007).

[11] D. Matyszewska and R. Bilewicz, Colloids Surf., A 321, 11 (2008).

[12] H.-J. Lehmler, W. Xie, G. D. Bothun, P. M. Bummer, and B. L. Knutson, Colloids Surf., B 51, 25 (2006).

[13] W. Xie, G. D. Bothun, and H.-J. Lehmler, Chem. Phys. Lipids 163, 300 (2010).

[14] W. Xie, I. Kania-Korwel, P. M. Bummer, and H.-J. Lehmler, Biochim. Biophys. Acta 1768, 1299 (2007).
[15] W. Xie, G. Ludewig, K. Wang, and H.-J. Lehmler, Colloids Surf., B 76, 128 (2010).

[16] L. R. Arriaga, I. Lopez-Montero, G. Orts-Gil, B. Farago, T. Hellweg, and F. Monroy, Phys. Rev. E 80, 031908 (2009).

[17] L. R. Arriaga, I. Lopez-Montero, F. Monroy, G. Orts-Gil, B. Farago, and T. Hellweg, Biophys. J. 96, 3629 (2009).

[18] L. R. Arriaga, R. Rodriguez-Garcia, I. Lopez-Montero, B. Farago, T. Hellweg, and F. Monroy, Eur. Phys. J. E 31, 105 (2010).

[19] B. Brüning, R. Stehle, P. Falus, and B. Farago, Eur. Phys. J. E 36, 77 (2013).

[20] M. Mell, L. H. Moleiro, Y. Hertle, P. Fouquet, R. Schweins, I. Lopez-Montero, T. Hellweg, and F. Monroy, Eur. Phys. J. E 36, 75 (2013).

[21] M. Hirai, R. Kimura, K. Takeuchi, M. Sugiyama, K. Kasahara, N. Ohta, B. Farago, A. Stadler, and G. Zaccai, Eur. Phys. J. E 36, 74 (2013).

[22] F. Mezei, Neutron Spin Echo (Springer, Berlin, 1980).

[23] B. Farago, Curr. Opin. Colloid Interface Sci. 14, 391 (2009).

[24] E. Evans and A. Yeung, Chem. Phys. Lipids 73, 39 (1994).

[25] U. Seifert and S. A. Langer, Europhys. Lett. 23, 71 (1993).

[26] W. Helfrich, Z. Naturforsch. C 28, 693 (1973).

[27] S. T. Milner and S. A. Safran, Phys. Rev. A 36, 4371 (1987).

[28] A. G. Zilman and R. Granek, Phys. Rev. Lett. 77, 4788 (1996).

[29] A. G. Zilman and R. Granek, Chem. Phys. 284, 195 (2002). 
[30] E. G. Brandt and O. Edholm, J. Chem. Phys. 133, 115101 (2010).

[31] K. R. Mecke, T. Charitat, and F. Graner, Langmuir 19, 2080 (2003).

[32] M. C. Rheinstädter, W. Häußler, and T. Salditt, Phys. Rev. Lett. 97, 048103 (2006).

[33] C. R. Safinya, E. B. Sirota, D. Roux, and G. S. Smith, Phys. Rev. Lett. 62, 1134 (1989).
[34] B. Brüning, E. Wald, W. Schrader, R. Behrends, and U. Kaatze, Soft Matter 5, 3340 (2009).

[35] T. Heimburg, Thermal Biophysics of Membranes (Wiley-VCH, Weinheim, 2007)

[36] J. Huang and G. W. Feigenson, Biophys. J 76, 2142 (1999).

[37] H. Nakahara, M. P. Krafft, A. Shibata, and O. Shibata, Soft Matter 7, 7325 (2011). 